\title{
Evaluation of Serological Tests Detecting Chlamydophila pneumoniae-specific Immunoglobulin M Antibody
}

\author{
Naoyuki Miyashita ${ }^{1}$, Yasushi Obase ${ }^{1}$, Minoru Fukuda ${ }^{1}$, Hisashi Shoji ${ }^{1}$, Keiji Mouri ${ }^{1}$, \\ Shinichi Yagi ${ }^{1}$, Koichiro Yoshida ${ }^{1}$, Kazunobu Ouchi ${ }^{2}$ and Mikio Oka ${ }^{1}$
}

\begin{abstract}
Object. To evaluate a newly developed enzyme-linked immunosorbent assay (ELISA) (Hitazyme C. pneumoniae) detecting Chlamydophila pneumoniae-specific immunoglobulin M (IgM) antibody, we compared the assay with culturing, immunoblotting and the microimmunofluorescence (MIF) test.

Patients and Methods. Two hundred five patients with stable chronic lung diseases without acute respiratory tract infections (ARTIs) and 116 healthy volunteers without ARTIs were enrolled in this study. Nasopharyngeal swab specimens and sera were obtained from all subjects for isolation and serological testing of $C$. pneumoniae.

Results. C. pneumoniae IgM-positive results were observed in $16.5 \%$ of patients with stable chronic lung diseases and in $8.6 \%$ of asymptomatic healthy subjects. However, there were no positive cases with cell culture, immunoblot or MIF test. In addition, no cases with a significant increase in IgA or IgG antibody titer for the ELISA kit and MIF test between paired sera were observed in the followed-up groups. IgM-positive cases were more frequent among patients with chronic obstructive pulmonary disease $(p=0.1566)$, collagen disease-associated interstitial lung disease $(p<0.0001)$ and cryptogenic organizing pneumonia $(p=0.0199)$ than among the healthy subjects.

Conclusion. Our results indicate that IgM-positive results with the ELISA kit do not always reflect acute $C$. pneumoniae infections. Further studies are needed, to determine an appropriate cut-off level and the possible causes of the false-positive results in the ELISA kit, such as other underlying conditions.
\end{abstract}

Key words: Chlamydophila pneumoniae, serology, immunoglobulin M, enzyme-linked immunosorbent assay, microimmunofluorescence test, false-positive

(DOI: 10.2169/internalmedicine.45.6074)

\section{Introduction}

Chlamydophila pneumoniae is a major pathogen in respiratory infections (1-6). It is involved in a wide spectrum of various respiratory tract infections in both immunocompetent and immunocompromised hosts. Several studies have suggested possible association of $C$. pneumoniae infection and acute exacerbation of asthma and chronic obstructive pulmonary disease (COPD) (1-4). Current detection methods of $C$. pneumoniae include culturing, antigen detection assays (the direct fluorescent antibody assay and enzyme immu- noassay), serology and molecular techniques $(1,7)$. Since cell cultures detecting $C$. pneumoniae require specialized laboratories and are expensive, time-consuming and laborintensive, the clinical usefulness is quite limited. Antigen detection assays are of limited sensitivity and specificity. Nucleic acid amplification techniques, such as the polymerase chain reaction, are highly sensitive techniques and have demonstrated sensitivity and specificity equal to or even better than those of conventional microbiologic tests. However, there are no available kits commercially, and only laboratory tests are used. The routine diagnostic method of $C$. pneumoniae is currently still serological analysis.

\footnotetext{
${ }^{1}$ Division of Respiratory Diseases, Department of Medicine, Kawasaki Medical School and ${ }^{2}$ Department of Pediatrics, Kawasaki Medical School, Kurashiki, Okayama

Received for publication June 10, 2006; Accepted for publication July 9, 2006

Correspondence to Dr. Naoyuki Miyashita, Division of Respiratory Diseases, Department of Medicine, Kawasaki Medical School, 577 Matsushima, Kurashiki, Okayama 701-0192
} 
Table 1. Prevalence of C. pneumoniae IgM Antibody in Different Underlying Lung Diseases

\begin{tabular}{lcc}
\hline Underlying disease & No. of patients & No. of positive (\%) \\
\hline Chronic obstructive pulmonary disease & 42 & $7(16.6)$ \\
Asthma & 34 & $3(8.8)$ \\
Nontuberculous mycobacteria & 33 & $4(12.1)$ \\
Lung cancer & 19 & $2(10.5)$ \\
Bronchiectasis* & 19 & $2(10.5)$ \\
Collagen disease-associated interstitial lung disease** & 18 & $9(50.0)$ \\
Idiopathic pulmonary fibrosis & 13 & $1(7.6)$ \\
Cryptogenic organizing pneumonia & 11 & $4(36.3)$ \\
Pneumoconiosis & 10 & $1(10.0)$ \\
Sarcoidosis & 4 & 0 \\
Sino-bronchial syndrome & 2 & 1 \\
& & \\
\hline * Including localized bronchiectasis & & \\
** Including 7 rheumatoid arthritis, 7 systemic sclerosis, 3 systemic lupus erythematosus, and one \\
polymyositis/dermatomyositis.
\end{tabular}

In Japan, a commercial kit detecting $C$. pneumoniaespecific immunoglobulin $\mathrm{G}(\mathrm{IgG})$ and $\operatorname{IgA}$ antibodies using an enzyme-linked immunosorbent assay (ELISA) (Hitazyme C. pneumoniae; Hitachi Chemical Co., Tsukuba, Japan) has been developed and is widely used (8). Recently, this ELISA kit has been available for detection of the $C$. pneumoniae-specific IgM antibody, and rapid diagnosis of C. pneumoniae infection has been expected. However, this ELISA kit has not been properly evaluated yet, due to the high prevalence rates of IgM positivity in both children and adults with acute respiratory tract infections (ARTIs) as reported in this kit. Therefore, most clinicians have been confused in making a diagnosis of acute $C$. pneumoniae infection, when using the diagnostic criteria of the kit. In the present study, to evaluate the reliability of the kit, we compared it with culture, immunoblot and the microimmunofluorescence (MIF) test of the current gold standard for serological testing of $C$. pneumoniae infections worldwide $(1,7)$.

\section{Patients and Methods}

\section{Study population}

C. pneumoniae infection appears much more common in patients with chronic lung diseases than in age-matched controls $(2-4,6)$. Thus, we enrolled two populations, healthy subjects and patients with stable chronic lung diseases, in this study. The subjects were 205 patients with stable chronic lung diseases without ARTIs, consisting of 113 males and 92 females with mean age 67.6 years (range 21 to 90 ). The patients regularly visited the outpatient clinic at the Kawasaki Medical School Hospital, between June 2005 and May 2006. Their underlying lung diseases are listed in
Table 1. On the other hand, 116 healthy volunteers without ARTIs were selected during the study period. The healthy subjects consisted of 65 males and 51 females with mean age 66.5 years (range 21 to 82), which were matched with those of the patients. Subjects were excluded from the study if they reported a history of infection with C. pneumoniae, if they had taken antibiotics within the preceding two weeks before enrollment, or if they reported having had a clinical syndrome compatible with pharyngitis, sinusitis, bronchitis, or pneumonia within the preceding six months before enrollment. Nasopharyngeal swab specimens and sera were obtained from all subjects with informed consent; the study protocol was approved by the Ethics Committee at Kawasaki Medical School.

\section{Culture}

Nasopharyngeal swab specimens for culture were placed in a sucrose-phosphate-glutamate transport medium. Each swab specimen was sonicated and briefly centrifuged (900x $\mathrm{g}$ for $10 \mathrm{~min}$ ), and the supernatant was overlayed on confluent monolayers of HEp-2 cells grown on round coverslips (14-mm in diameter) set in a 24-well plastic cell culture plate. The plate was centrifuged at $1,200 \times \mathrm{g}$ for $60 \mathrm{~min}$ at room temperature. Next, one $\mathrm{mL}$ of a culture medium consisting of Eagle's minimal essential medium (Nissui Pharmaceuticals Co., Tokyo), 10\% heat-inactivated fetal calf serum (Gibco BRL Life Technologies Inc., Grand Island, NY, USA), and cycloheximide (Nakarai Tesque Inc., Tokyo, Japan) at a final concentration of $1 \mu \mathrm{g} / \mathrm{mL}$ was applied. Then, the plates were incubated in $5 \% \mathrm{CO}_{2}$ at $35^{\circ} \mathrm{C}$ for $72 \mathrm{~h}$, and all specimens were passed twice. Following incubation, a genus-specific fluorescein isothiocyanate-conjugated monoclonal antibody (Chlamydia FA Seiken; Denka Seiken, To- 


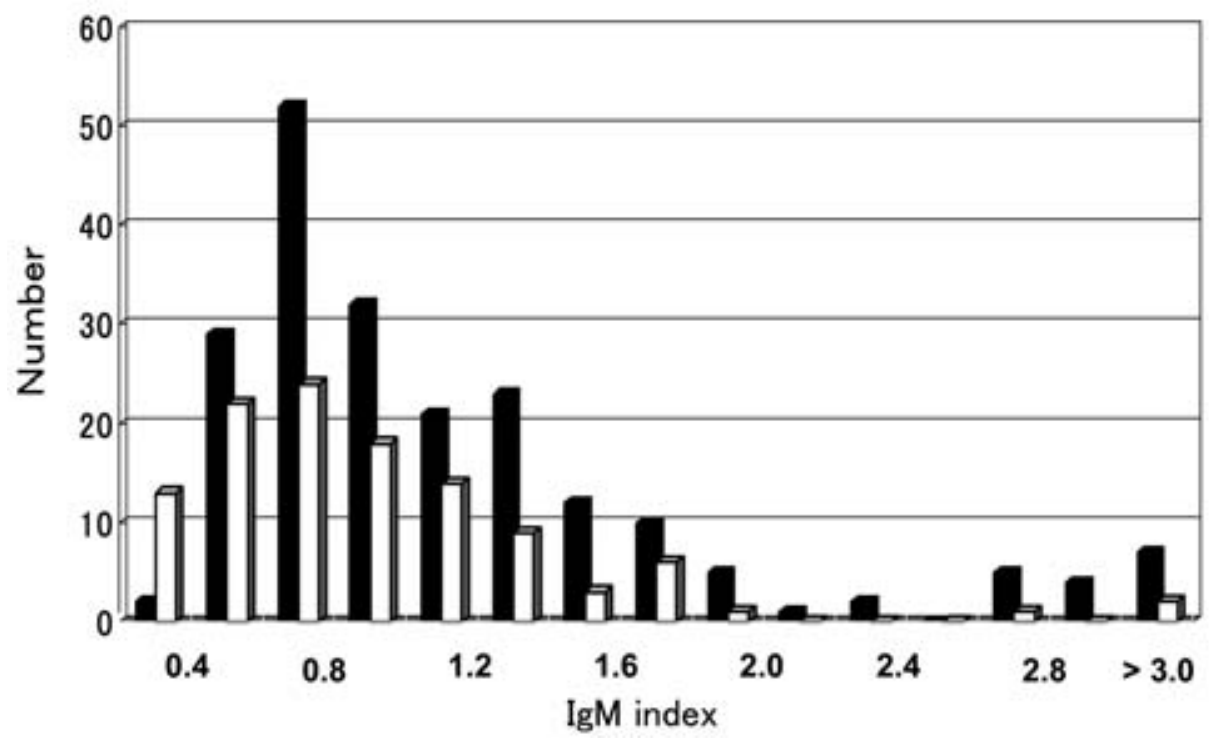

Figure 1. Distribution of IgM antibody titer to Chlamydophila pneumoniae using the ELISA kit, in 205 patients with stable chronic lung diseases (black bar) and 116 healthy controls (white bar).

kyo) and $C$. pneumoniae species-specific monoclonal antibodies were used to stain inclusions (9). Inclusions were observed with a Nikon epifluorescence microscope at $\times 200$ or $\times 400$ magnification.

\section{Serology}

The ELISA kit (Hitazyme C. pneumoniae) and the MIF test were used to measure the $\operatorname{IgM}, \operatorname{IgA}$ and/or $\operatorname{IgG}$ antibodies to $C$. pneumoniae. The ELISA detects antibodies to the chlamydial outer membrane complex excluding lipopolysaccharide, which was isolated from purified elementary bodies (EBs) of the C. pneumoniae YK-41 strain (10). The concentration of the IgM, IgA and IgG antibodies against C. pneumoniae in each sample were expressed as the $\operatorname{IgM}, \operatorname{IgA}$ or IgG index. The IgM, IgA or IgG index was determined by calculating the corrected optimal density (OD) at $405 \mathrm{~nm}$ of the each sample divided by the IgM, IgA or IgG cut-off value, as shown in the following formulas. The corrected OD of a sample=the sample's OD at $405 \mathrm{~nm} \times$ the reference value of the positive control/mean OD at $405 \mathrm{~nm}$ of the positive control. The cut-off value of $\mathrm{IgM}=$ mean $\mathrm{OD}$ at 405 $\mathrm{nm}$ of the negative control $\times$ the reference value of the positive control/the mean OD at $405 \mathrm{~nm}$ of the positive control +0.30 . Index values $\geqq 1.60$ are defined as positive for IgM antibody (11). The cut-off value of $\operatorname{IgA}$ or $\operatorname{IgG}=$ mean $\mathrm{OD}$ at $405 \mathrm{~nm}$ of the negative control $\times$ the reference value of the positive control/the mean OD at $405 \mathrm{~nm}$ of the positive control +0.20 . Index values $\geqq 1.10$ are defined as positive for $\operatorname{IgA}$ or $\operatorname{IgG}$ antibody (12). The increase of 1.00 in index for $\operatorname{IgA}$ and 1.35 for IgG between paired sera was considered to indicate an acute infection (12).

The MIF test was primarily developed for C. trachomatis by Wang and Grayston (13), but it was later adapted for use in serodiagnosis of $C$. pneumoniae infections (14). The EBs of the YK-41 and TW-183 strains were purified by a method of continuous Urografin (Schering AG, Berlin/ Bergkamen, Germany) gradient centrifugation (40-52\%), as described previously (15). The EBs were resuspended in a solution of $2 \%$ yolk sac in phosphate-buffered saline $(\mathrm{pH}$ 7.2) containing $0.02 \%$ formalin. The presence of $\operatorname{IgM}$ and IgG antibodies against C. pneumoniae was detected using commercial fluorescein isothiocyanate conjugated goat antihuman IgM and IgG (Medical and Biological Laboratories, Nagoya, Japan). Rheumatoid factor (RF) was absorbed with Gullsorb (Gull Laboratories, Salt Lake City, Utah, USA) before IgM titrations. An anti-C. pneumoniae antibody of titer $\geqq 1: 32$ for IgM or a four-fold increase in IgG was considered to indicate an acute infection. Positive sera in the ELISA kit and/or the MIF test were also analyzed by immunoblotting, as reported previously (16). Purified EBs of the YK-41 strain were employed as the antigen.

\section{Statistical analysis}

Statistical analysis was done by Fisher's Exact test. All $p$ values were two-sided, and $p<0.05$ was considered statistically significant.

\section{Results}

Distribution of the IgM index measured by the ELISA kit in the patients and healthy subjects is shown in Fig. 1. C. pneumoniae IgM-positive cases were observed in 34 $(16.5 \%)$ patients and $10(8.6 \%)$ healthy subjects. However, neither cell culturing nor the MIF test revealed any positive cases in the two groups. The IgM-positve cases with the ELISA kit were all negative with immunoblot analysis.

Table 1 shows the prevalence of IgM-positive with the ELISA kit in different underlying lung diseases. IgMpositive cases were more frequent among patients with COPD $(p=0.1566)$, collagen disease-associated interstitial 


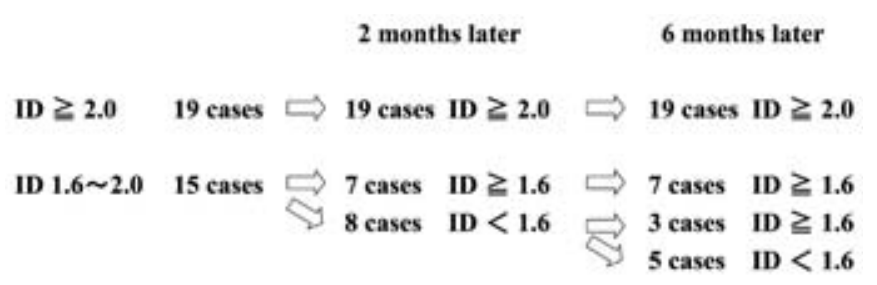

Figure 2. Change of IgM antibody titer to Chlamydophila pneumoniae in 34 IgM-positive cases using the ELISA kit, for six months.

lung disease (ILD) $(p<0.0001)$ and cryptogenic organizing pneumonia (COP) $(p=0.0199)$ than among the healthy subjects. Collagen disease-associated ILD included seven rheumatoid arthritis, seven systemic sclerosis, three systemic lupus erythematosus and one polymyositis/dermatomyositis. Among the patients with collagen disease-associated ILD, six patients with rheumatoid arthritis and three patients with systemic sclerosis were IgM-positive.

We followed-up 34 IgM-positive patients with the ELISA kit, and measured the IgM, IgA and IgG antibodies two and six months later (Fig. 2). In 19 cases with a moderate or more elevated IgM titer (index $>2.00$ ), all were still positive two and six months later. In 15 cases with mild elevation of the IgM titer (index 1.60-2.00), however, eight positives were changed to negative two months later. Of these eight cases, five were still negative and three returned to positive six months later. Of the seven cases with IgM-positivity two months later, all were still positive six months later. We also followed-up 20 patients who were initially IgM negative (index 1.20-1.59), of which eight were positive two months later. Of these eight cases, four were still positive and four returned to negative six months later. Neither the MIF test nor the immunoblot analysis revealed any IgM-positive cases in the two followed-up groups. In addition, no cases with significant increase in $\operatorname{IgA}$ or $\operatorname{IgG}$ antibody titer for the ELISA kit and MIF test between paired sera were observed in the two followed-up groups.

\section{Discussion}

Regarding serological tests for C. pneumoniae infections, only the MIF test is strongly recommended (7). However, most Japanese clinicians and researchers have not used the MIF test for routine diagnosis, because this assay is technically demanding, time-consuming, and difficult for antigen preparation and interpretation. Then, in Japan, a partially automated ELISA kit (Hitazyme C. pneumoniae) has been developed for the routine diagnosis, resulting in convenience for routine use (8). Recently, most clinicians and researchers have used this kit, but kits for only $\operatorname{IgG}$ and $\operatorname{IgA}$ antibodies have been available. Among the Ig classes, the IgM antibody is so helpful for rapid diagnosis of acute C. pneumoniae infections because of its early serologic response to $C$. pneumoniae rather than the $\operatorname{IgG}$ antibody $(1,7)$. In a clinical setting, a single rather than acute and convalescent samples are received for the diagnosis of acute infections. Recently, an ELISA kit for the IgM antibody has also become available, but this assay has not been properly evaluated yet, as compared with the MIF test and immunoblotting.

In this study, the ELISA kit was prospectively evaluated in healthy controls and patients with stable chronic lung diseases. The ten $(8.6 \%)$ healthy subjects and 34 (16.5\%) patients had the positive criteria in the ELISA kit, but there were no positive cases for the MIF test or cultures. In addition, no positive cases for immunoblot were observed in the IgM-positive cases for the ELISA kit. These results indicate that the IgM positivity with the ELISA kit does not always reflect acute $C$. pneumoniae infections and probably includes false-positive findings.

We followed up all of the IgM-positive and some IgMnegative cases (index 1.20-1.59) with the ELISA kit, for six months. Some cases with mild elevation of IgM titer (index 1.60-2.00) were negative two months later, while some returned to positive again six months later. Some IgMnegative (index 1.20-1.59) cases showed positivity two months later, but some returned to negative again six months later. However, neither the MIF test nor the immunoblot analysis revealed any IgM-positive cases in the two follow-up groups. Thus, these results strongly suggest that the $\operatorname{IgM}$ titer for the ELISA kit around the cut-off level (index 1.20-2.00) is unreliable as a diagnostic criterion, at the present, and the cut-off level should be changed. The present results suggest that if index values $\geqq 5.0$ are defined as positive for IgM antibody, the diagnostic criteria is relatively reliable.

$\mathrm{RF}$, an autoantibody that reacts with antigenic sites located in the $\mathrm{Fc}$ region of $\mathrm{IgG}$, sometimes causes falsepositive IgM for $C$. pneumoniae $(1,17)$, and therefore, a RF-absorbing reagent is used in the MIF test. This RFabsorbing reagent is also used in the ELISA kit, and no cross-reactions have been confirmed when the RF level is less than $500 \mathrm{IU} / \mathrm{mL}$. In the present study, RF was measured in all of the IgM-positive subjects to exclude false-positive results, and only one IgM-positive subject had a high RF level $(1,680 \mathrm{IU} / \mathrm{mL})$, whereas the others had low or negative RF levels. Here, the prevalence of IgM-positive cases was higher in patients with chronic lung diseases than in healthy controls. Thus, underlying diseases such as COPD, collagen disease-associated ILD and COP, may cause false-positive results for the IgM antibody with the ELISA kit. To determine the possible causes of the false-positive results in the ELISA kit, such as other underlying conditions, further studies are needed.

Hermann and coworkers evaluated 11 commercial serological tests including the ELISA kit used in the present study (Hitazyme C. pneumoniae "Elegance", commercially available in Europe and Asia) for IgG antibodies in healthy population (18). Their sensitivities were widely dispersed, probably depending on the kind and the quality of the antigen preparation. The sensitivities and specificities of the 
MIF tests were high, but a poor correlation was found for the results of the Elegance in comparison to those of the MIF test and other serological tests (18). Then, they indicated that Elegance might be not useful for the investigation of the seroprevalence of $C$. pneumoniae in healthy population. For IgM antibodies, in the present study, no positive correlations were observed between the ELISA kit and MIF test or immunoblot in either the healthy controls or in the patients with stable chronic lung diseases. Considered together with our data, these results indicate that the discrepancy may be explained by the different cut-off levels of the tests.

In conclusion, a newly developed ELISA kit (Hitazyme
C. pneumoniae) to detect the C. pneumoniae-specific IgM antibody frequently shows false-positive findings at the current cut-off level. In addition to the fact that RF is a wellknown false-positive factor, chronic lung diseases such as COPD, collagen diseases-associated ILD and COP may cause false-positive IgM results in this ELISA kit. To determine an appropriate cut-off level for the ELISA kit, further clinical studies are necessary.

This work was supported by the 32nd Kanae Foundation for Life \& Socio-Medical Science, and Project Research Grants from Kawasaki Medical School (13-401, 14-402, 15-405A, 16-405M, 17-402M, 18-401).

\section{References}

1. Kuo CC, Jackson LA, Campbell LA, Grayston JT. Chlamydia pneumoniae (TWAR). Clin Microbiol Rev 8: 451-461, 1995.

2. Blasi F. Atypical pathogens and respiratory tract infections. Eur Respir J 24: 171-181, 2004.

3. Miyashita N, Kubota Y, Nakajima M, Niki Y, Kawane H, Matsushima T. Chlamydia pneumoniae and exacerbations of asthma in adults. Ann Allergy Asthma Immunol 80: 405-409, 1998.

4. Miyashita N, Nakajima M, Niki Y, Kawane H, Matsushima T. Chlamydia pneumoniae infection in patients with diffuse panbronchiolitis and COPD. Chest 114: 969-971, 1998.

5. Miyashita N, Fukano H, Mouri K, et al. Community-acquired pneumonia in Japan: a prospective ambulatory and hospitalized patient study. J Med Microbiol 54: 395-400, 2005.

6. Fujita J, Bandoh S, Tokuda M, et al. Non-specific interstitial pneumonia and Chlamydia pneumoniae infection. Jpn J Infect Dis 54: $225-228$,

7. Dowell SF, Peeling RW, Boman J, et al. Standardizing Chlamydia pneumoniae assays: recommendations from the Centers for Disease Control and Prevention (USA) and the Laboratory Centre for Disease Control (Canada). Clin Infect Dis 33: 492-503, 2001.

8. Kishimoto T, Kubota Y, Matsushima T, et al. Assay of specific anti-Chlamydia pneumoniae antibodies by ELISA method 1 . Evaluation of ELISA kit using outer membrane complex. Kansenshogaku Zasshi 70: 821-829, 1996 (in Japanese).

9. Miyashita N, Fukano H, Okimoto N, et al. Clinical presentation of community-acquired Chlamydia pneumoniae pneumonia in adults. Chest 121: 1776-1781, 2002.

10. Miyashita N, Kanamoto Y, Matsumoto A. The morphology of Chlamydia pneumonaie. J Med Microbiol 38: 418-425, 1993.

11. Kishimoto T, Ogawa M, Shiga S, Yamazaki T, Matsushima T. Setting of serological criteria for specific IgM antibody to C. pneu- moniae by Hitazyme $C$. pneumoniae in adults. Kansenshogaku Zasshi 75: 762, 2001 (in Japanese).

12. Kishimoto T, Matsushima T, Morikawa T, Kawagoe K. Assay of specific anti-Chlamydia pneumoniae antibodies by ELISA method. 3. Setting of serological criteria. Kansenshogaku Zasshi 73: 457466, 1999 (in Japanese).

13. Wang SP, Grayston JT. Immunologic relationship between genital TRIC, lymphogranuloma venereum, and related organisms in a new microtiter indirect immunofluorescence test. Am J Ophthalmol 70: 367-374, 1970.

14. Wang SP. The microimmunofluorescence test for Chlamydia pneumoniae infection: technique and interpretation. J Infect Dis 181: S421-S425, 2000.

15. Miyashita N, Matsumoto A. Establishment of a particle-counting method for purified elementary bodies of Chlamydiae and evaluation of sensitivities of IDEIA Chlamydia kit and DNA probe by using the purified elementary bodies. J Clin Microbiol 30: 29112916, 1992.

16. Miyashita N, Matsumoto A, Kubota Y, Nakajima M, Niki Y, Matushima T. Continuous isolation and characterization of Chlamydia pneumonaie from a patient with diffuse panbronchiolitis. Microbiol Immunol 40: 547-552, 1996.

17. Verkooyen RP, Hazenberg MA, Van Haaren GH, et al. Age-related interference with Chlamydia pneumoniae microimmunofluorescence serology due to circulating rheumatoid factor. J Clin Microbiol 30: 1287-1290, 1992.

18. Hermann C, Graf K, Groh A, Straube E, Hartung T. Comparison of eleven commercial tests for Chlamydia pneumoniae-specific immunoglobulin $\mathrm{G}$ in asymptomatic healthy individuals. J Clin Microbiol 40: 1603-1609, 2002.

(C) 2006 The Japanese Society of Internal Medicine http://www.naika.or.jp/imindex.html 